\title{
Tweeting PP: an analysis of the 2015-2016 Planned Parenthood controversy on Twitter
}

\author{
Leo Han ${ }^{\mathrm{a},{ }^{\star}, \text { Lisa Han }}{ }^{\mathrm{b}}$, Blair Darney ${ }^{\mathrm{a}, \mathrm{c}}$, and Maria I. Rodriguez ${ }^{\mathrm{a}}$ \\ aOregon Health \& Science University, Department of Obstetrics and Gynecology, Portland, OR \\ bUniversity of California, Santa Barbara, Department of Film and Media Studies, Santa Barbara, \\ CA \\ ${ }^{\circ}$ Center for Health Systems Research, National Institute of Public Health, Cuernavaca, Mexico
}

\begin{abstract}
Objectives-We analyzed Twitter tweets and Twitter-provided user data to give geographical, temporal and content insight into the use of social media in the Planned Parenthood video controversy.

Methodology-We randomly sampled the full Twitter repository (also known as the Firehose) $(n=30,000)$ for tweets containing the phrase "planned parenthood" as well as group-defining hashtags "\#defundpp" and "\#standwithpp." We used demographic content provided by the user and word analysis to generate charts, maps and timeline visualizations. Chi-square and $t$ tests were used to compare differences in content, statistical references and dissemination strategies.
\end{abstract}

Results-From July 14, 2015, to January 30, 2016, 1,364,131 and 795,791 tweets contained "\#defundpp" and "\#standwithpp," respectively. Geographically, \#defundpp and \#standwithpp were disproportionally distributed to the US South and West, respectively. Word analysis found that early tweets predominantly used "sensational" words and that the proportion of "political" and "call to action" words increased over time. Scatterplots revealed that \#standwithpp tweets were clustered and episodic compared to \#defundpp. \#standwithpp users were more likely to be female [odds ratio (OR) 2.2, confidence interval (CI) 2.0-2.4] and have fewer followers (median 544 vs. 1578, $\mathrm{p}<.0001)$. \#standwithpp and \#defundpp did not differ significantly in their usage of data in tweets. \#defundpp users were more likely to link to websites (OR 1.8, CI 1.7-1.9) and to other online dialogs (mean 3.3 vs. $2.0 \mathrm{p}<.0001$ ).

Conclusion-Social media analysis can be used to characterize and understand the content, tempo and location of abortion-related messages in today's public spheres. Further research may inform proabortion efforts in terms of how information can be more effectively conveyed to the public.

Implications-This study has implications for how the medical community interfaces with the public with regards to abortion. It highlights how social media are actively exploited instruments for information and message dissemination. Researchers, providers and advocates should be monitoring social media and addressing the public through these modern channels.

*Corresponding author. Tel.: +1 720320 9555. hanl@ohsu.edu (L. Han). 


\section{Keywords}

Planned Parenthood; Social media; Twitter; Abortion

\section{Introduction}

On July 14th, 2015, the Center for Medical Progress, an antiabortion organization posing as a biotechnology firm, began releasing videos online of Planned Parenthood employees discussing the purchase of fetal tissue for research purposes [1]. These videos contained surreptitiously recorded conversations that were edited to imply misconduct on the part of Planned Parenthood. Although the videos were discredited [2], they gained widespread media coverage and ignited public debate over abortion care and research with fetal tissue in the United States (US). A significant political response ensued, including congressional hearings, legislative attempts to make Planned Parenthood ineligible for federal funding and strong antiabortion rhetoric from presidential candidates. Social media platforms such as Twitter, Facebook and Instagram both reflected and influenced the public discourse of this controversy $[3,4]$.

Twitter is a free microblogging media tool that forms a valuable repository of publically messaged opinions [5-7]. Approximately one quarter of adults online use Twitter [8]. Users are able to post messages of 140 characters (called a "tweet") to either the general public or a group of followers, who subscribe to posts from individual users. While companies often use Twitter to track consumer trends, its data repository of over 310 million monthly users is also now used by health researchers [9]. Related work has included the use of Twitter as a predictive tool for epidemiological spread of disease, measurement of public sentiment on health policy changes and even exploration of using social media language analysis to prospectively follow patient health [10-12].

While much of the interest in Twitter centers around monitoring ambient discussions in the public sphere, users employ Twitter to strategically spread messages and information in order to influence others [13-15]. Twitter employs several platform functions that increase visibility and interest in tweets. Hashtags (“\#”) assigned to keywords express affiliations to various digital social publics and dynamic topic themes ("threads") $[16,17]$. Users can also repost a previously tweeted post of another user from their own account, which is known as a "retweet" or direct tweets at other specific users by including their username in the tweet ("mentions"). These both allow messages to be amplified through a new set of followers. Finally, because tweets are limited in characters, they often link to additional websites with more content including blogs, news articles, images and videos.

Abortion is one of the most frequently discussed medical procedures on social media platforms [18]. In the case of the Planned Parenthood video controversy, users took to Twitter with two very opposite intentions of influencing the discussion of abortion [19]. This public debate had political, financial, medical and legal consequences for abortion provision. The objective of our research was to describe the public discourse on this controversy in terms of both content being shared and strategies used to disseminate information on Twitter. We compared the demographic and content characteristics of tweets from the group-defining 
hashtags "\#defundpp" and "\#standwithpp" to obtain novel insight into the public debate on abortion.

\section{Methods}

We conducted a mixed-methods, retrospective, repeated cross-sectional study using Twitter data between July 14, 2015, and January 30, 2016, a 180-day span that begins with release of the first video and concludes just after the indictment of two employees from the Center for Medical Progress [2]. We used both qualitative and quantitative methods to compare messaging around this issue among proabortion (represented by \#standwithpp) and antiabortion (represented by \#defundpp) advocates. We chose these two hashtag campaigns in particular because they were both the most trending hashtags used at the time as well as championed by the major advocacy groups in question. \#standwithpp was used and continues to be used directly by Planned Parenthood, major news outlets (CNN, Newsweek, Mic and Bustle, among others), politicians and celebrity advocates. While antiabortion users tweeted a variety of hashtags, \#defundpp was used by legislators and public figures and has headlined anti-Planned Parenthood rallies.

Our study was deemed exempt by the Oregon Health \& Science University institutional review board.

\subsection{Database}

We collected data using Crimson Hexagon (Crimson Hexagon, Boston, MA, USA; 2016), a licensed social media analytic tool that samples the entire collection of tweets on Twitter, known as the Firehose, with unbiased and statistically consistent categorical proportions that is available for an institutionally negotiated fee [8,20]. During our inquiry period, a total of 6,884,998 tweets contained the phrase "planned parenthood." Also during this time, 1,364,131 tweets contained an antiabortion message using the hashtag "\#defundpp," and 795,791 tweets contained a proabortion stance utilizing the hashtag "\#standwithpp." For each of these phrases, we sampled 10,000 random tweets over this time period.

\subsection{Variables}

For each tweet, Twitter provides certain information related to the user account of the tweet (Table 1). Additionally, if provided by the user, Crimson Hexagon can also give location data associated with the Twitter account including country, state, and city or urban area; the author's gender; and the author's Klout score. Klout score is a validated, industry-accepted measure of "social influence" based on the author's presence and follower count across several social media platforms [21,22]. Klout scores have a range of 1-100, with higher scores corresponding to greater strength and spread of influence.

We uploaded our tweet sample to the open web app Visage (Column Five, Irvine, CA, USA; 2016) to create topic graphs that helped us understand the most prevalent themes in the tweets. The tweets were then queried for the most frequently mentioned words and categorically coded based on the three themes that dominated the conversation: "political," "action" and "sensational." "Political" words were defined by any association with a political institution or movement (i.e., hillaryclinton, republican). The "action" category 
included verbs calling for a particular activity (i.e., support, donate). Finally, "sensational" terms were defined as such because they employed dramatic or visceral imagery (i.e., chop, murder, war). Neutral, descriptive words such as "planned parenthood" or "with" were excluded. In order to limit researcher bias in categorizing these terms, two investigators independently coded the 200 most common words. In the event of a disagreement, a third investigator arbitrated the final category. Table 2 contains an example of list of the top 40 most tweeted words and their category designations.

We also wanted to examine the use of statistical or data-based arguments. We found that use of the "\%" symbol was almost always associated with statistical statements such as " $87 \%$ of Planned Parenthood revenue comes from abortions!" We also assessed for use of platformspecific messaging strategies including retweets, links to external websites, "mentions" of other users (excluding retweets) and association with other threads (additional hashtags "\#”). For geographical categorization, we adapted preexisting US census regions (West, Midwest, South and Northeast) [23].

\subsection{Analysis}

We analyzed the output through a variety of qualitative and quantitative techniques in order to characterize the origin, content and cadence of the conversation. We used tabulations, descriptive statistics and graphs to examine sociodemographic variables and tweet content. Chi-square and $t$ tests were used to compare differences in content, statistical references and dissemination strategies by hashtag (indicating proabortion vs. antiabortion affiliations). We generated repeated cross-sectional geographic plots and scatterplots over time using RAW, an open web app that creates custom vector-based visualizations using the D3.js JavaScript library (Density Designs, Milan, Italy; 2016). Additionally, because tweeting is highly correlated with real-time information, these were examined in relationship to known significant events that occurred during this time period (e.g., Republican Presidential Primary debates, shooting at the Colorado Springs Planned Parenthood). We used Stata 13.0 (StataCorp LP, College Station, TX, USA; 2013) and Microsoft Excel 14.4.3 (Microsoft Corporation, Redmond, WA, USA; 2010) for analysis.

\section{Results}

Our final data set utilized 30,000 tweets from 18,869 users. We first examined specific author characteristics to identify differences in location, gender and social influence by hashtag used. Fig. 1 depicts the geographical distribution of \#defundpp and \#standwithpp tweets. \#defundpp tweets were much more likely to occur in the American South (44\% vs. $29, \mathrm{p}<.001$ ), while \#standwithpp was seen disproportionately in the American West (26\% vs. $19 \%, \mathrm{p}<.001$ ) and Northeast (19\% vs. 12\%, p<.001). Equal proportions of \#defundpp and \#standwithpp tweets came out of the Midwest (17\%). Hashtag use was also differentiated by gender. \#standwithpp tweets were predominately female ( $66 \%$ vs. $34 \%, \mathrm{p}<.001$ ), while \#defundpp tweets were more likely to be male (53\% vs. $47 \%$, p<.001). Moreover, \#defundpp users had significantly higher social influence (mean Klout score 47.8 vs. 41.4, p<.001) and more followers of their accounts (median 1578 vs. 544, p<.001). 
We compared messaging tactics between \#defundpp and \#standwithpp campaigns by looking at both the content of tweets as well as strategies used to amplify messaging. There was no significant difference in use of statistical arguments (1\% vs. .9\%, $\mathrm{p}=.385)$. Our word analysis using coded designations also demonstrated differences in the \#stand-withpp and \#defundpp content. For the general thread "planned parenthood," $15.8 \%$ of words were categorized as "political," 9.7\% as "action" and 11.4\% as "sensational." For \#defundpp specific tweets, we found that of the most common words, $36.9 \%$ of tweets were "political," $11.1 \%$ were "action" and $19.2 \%$ were "sensational." Meanwhile, for \#standwithpp, $23.4 \%$ were "political," $15.8 \%$ "action" and 5.1\% "sensational." We analyzed how the use of these words evolved over time in the public discourse (Fig. 2). While initial tweets predominantly used "sensational" words, over time, the proportion of politically associated tweets or "action" tweets increased.

Twitter-specific messaging strategies, such as including external links, mentions, retweeting and use of additional hashtags, are used to amplify messaging on social media. We examined whether \#standwithpp and \#defundpp tweets used these techniques differently. We found that \#defundpp users were significantly more likely to insert external links (83\% vs. 78\%, $\mathrm{p}<.001)$, mention another user ( $40 \%$ vs. $37 \%, \mathrm{p}<.05)$, as well as retweet previous user comments $(86 \%$ vs. $65 \%, \mathrm{p}<.001)$. \#defundpp users were also much more likely to use additional hashtags to link their tweets to other threads (mean hashtags 3.3 vs. $2.0, \mathrm{p}<.001$ ).

Finally, we created various scatterplots over time to better understand the relationship of our demographic variables with tweet output and significant events. Fig. 3 is a scatterplot of \#standwithpp and \#defundpp tweets plotted over time with significant events marked on the timeline. Visually, it appeared that the \#standwithpp tweets were episodic over this time and highly clustered around major events. \#defundpp tweets appeared to have more consistent output over the study period. To quantify this, we compared tweets that occurred within 1 week of these events and found that \#standwithpp tweets were far more likely to occur during this window (55\% vs. $39 \%, \mathrm{p}<.001)$.

\section{Comment}

Social media platforms such as Twitter play an important role in information dissemination, idea amplification and digital reflection of public sentiment [5,24,25]. In this study, we provide an analysis of public discourse on abortion and fetal tissue research controversy on Twitter. Important differences were identified in both the content shared by users and the methods used to disseminate messaging by pro- and antiabortion advocates. There were also significant demographic differences in geography, gender and social media engagement.

This study also provides one of the first attempts to formally analyze tweet content by examining messaging tactics using platform functions and word analysis. We found that antiabortion tweets appeared to use more dissemination strategies such as co-hashtagging mentions and retweeting. We were also able to use Twitter to monitor the evolution of this debate over a period of time. Our findings suggest that the Planned Parenthood debate, while originally fanned by the imagery implied in the videos, evolved as a political sound bite with increasing mentions in the context of politics and political action. 
Our study shows that social media reflect many of the attitudes and opinions animating the public discourse of abortion provision. Previous research focusing on political events pertaining to abortion also found a robust public response on Twitter [26]. While our analysis similarly focuses on a period of significant controversy and public attention, it provides some new insight into the greater abortion discourse as a whole. Because Planned Parenthood is abortion's most visible US institution, we believe that it is fair to extrapolate anti-Planned Parenthood sentiment as antiabortion sentiment and, similarly, pro-Planned Parenthood postings as reflective of a proabortion sentiment. It has been shown that personal perspectives on social media leanings are often heavily biased by self-selecting social circles and algorithmically curated media feeds to match the readers' opinions [27,28]. As a result, without separate research inquiry, it may not be evident to many people that antiabortion posts dominated this discussion almost 2:1 during this period or that these posts came from more "influential" users. Similarly, it may be surprising that while the large body of evidence about the safety of abortion supports its unregulated provision [29,30], both sides appeared to employ statistical arguments with equal frequency.

There are significant limitations to this analysis. An inherent limitation to any analysis of a social media platform is that there will be population biases reflective of the platform's user base [31]. Twitter users tend to be younger, better educated, wealthier and more ethnically diverse [32]. Second, our study is limited by the types of data available from the Twitter Firehose. This data set is not constructed for public health research. Thus, key demographic information is not available or is restricted to self-report (gender and location), limiting our interpretation. Furthermore, if self-reported data are systematically missing, this can bias results. Third, hashtag analysis carries inherent biases that can skew data because hashtags themselves are user-defined surrogates. There may be some classification errors (for example, it is possible that some users are linking tweets to hashtags of the opposite sentiment to influence or infiltrate that dialog), and tweeting trends in these hashtag samples may not be externally valid to larger populations [33,34]. Fourth, in addition to words, tweets often contain images or links. These are important pieces of content that at this point we are unable to analyze. Fifth, this analysis is cross-sectional to a specific period of highvolume social media engagement around this topic. Results during this time should not be extrapolated to subsequent time periods. Finally, while we provided unique information about what is contained in the discourse, we cannot measure the impact of the message. There are some platform outcomes such as "likes" that hold potential as surrogates, but further research is needed to develop mechanisms for extracting these data. However, our study is one of the first attempts to examine a social media data set for abortion-related content using both quantitative and qualitative methods. We believe that our analysis significantly develops a nascent field.

There is important insight to be gained from studying medical issues like abortion on social media. We are increasingly aware that scientific data siloed to conventional medical journals may have little impact on public knowledge or perception [35]. In other recent examples such as transgender bathroom laws and gun violence, we have seen that public discourse can lead to political reactions and consequences [36,37]. Information regarding abortion is easily and often distorted, and distorted information may be the first information received by the public at large [38]. Research on the presence of distorted medical information on social 
platforms provides some insight about what the public is actually seeing and saying. Moreover, analysis such as this may inform proabortion efforts by describing how information can be effectively conveyed to the public.

As the interface of transmitting medical information happens increasingly outside the medical office and in the digital world, medical research needs to expand outside of traditional media to influence these spheres. Public discourse can be considered an important outcome for measuring the impact of research, particularly research on abortion and reproductive health. We hope that our study will encourage further rigorous, multidisciplinary inquiry into this field.

\section{Acknowledgments}

\section{Funding}

Darney is partially supported by a Society of Family Planning Junior Investigator Award (SFPRF9-JI2; Darney, PI). Dr. Rodriguez is a Women's Reproductive Health Research fellow; grant 1K12HD085809.

\section{References}

1. Calmes, J. Video accuses Planned Parenthood of crime. n.d. http://www.nytimes.com/ 2015/07/15/us/video-accuses-planned-parenthood-of-crime.html (accessed November 23, 2016)

2. 2 Abortion foes behind Planned Parenthood videos are indicted - the New York Times. n.d. http:// www.nytimes.com/2016/01/26/us/2-abortion-foes-behind-planned-parenthood-videos-areindicted.html (accessed July 25, 2016)

3. Lauren Hudel, Goodman. Planned Parenthood in crisis: social media strategies and frames. master's thesis. 2016:2836.

4. Garimella, K., De Francisci Morales, G., Gionis, A., Mathioudakis, M. Proc ninth ACM Int Conf Web search data min. ACM; 2016. Quantifying controversy in social media; p. 33-42.

5. O'Connor B, Balasubramanyan R, Routledge BR, Smith NA. From tweets to polls: linking text sentiment to public opinion time series. ICWSM. 2010; 11:1-2.

6. Manovich, L. Trending: the promises and the challenges of big social data. Manovich. 2011. http:// www.manovich.net (accessed June 2, 2017)

7. Papacharissi Z. The virtual sphere the internet as a public sphere. New Media Soc. 2002; 4:9-27.

8. Street 1615 L., NW, Washington S 800, inquiries D 200362024194300 | M 2024194349 | F 202 4194372 |M. Twitter demographics. Pew res cent internet Sci tech. 2015. http:// www.pewinternet.org/2015/08/19/mobile-messaging-and-social-media-2015/2015-08-19_socialmedia-update_11/ (accessed May 11, 2016)

9. Company | about. n.d. https://about.twitter.com/company (accessed May 11, 2016)

10. Signorini A, Segre AM, Polgreen PM. The use of Twitter to track levels of disease activity and public concern in the U.S. during the influenza a H1N1 pandemic. PLoS One. 2011; 6:e19467. http://dx.doi.org/10.1371/journal.pone.0019467. [PubMed: 21573238]

11. Wong CA, Sap M, Schwartz A, Town R, Baker T, Ungar L, et al. Twitter sentiment predicts affordable care act marketplace enrollment. J Med Internet Res. 2015; 17 http://dx.doi.org/ 10.2196/jmir.3812.

12. Padrez, KA., Ungar, L., Schwartz, HA., Smith, RJ., Hill, S., Antanavicius, T., et al. Linking social media and medical record data: a study of adults presenting to an academic, urban emergency department. BMJ Qual Saf. 2015. http://dx.doi.org/10.1136/bmjqs-2015-004489

13. Bond RM, Fariss CJ, Jones JJ, Kramer ADI, Marlow C, Settle JE, et al. A 61-million-person experiment in social influence and political mobilization. Nature. 2012; 489:295-8. http:// dx.doi.org/10.1038/nature11421. [PubMed: 22972300] 
14. Romero, DM., Galuba, W., Asur, S., Huberman, BA. Jt Eur Conf Mach Learn Knowl Discov Databases. Springer; 2011. Influence and passivity in social media; p. 18-33.

15. Boulianne S. Social media use and participation: a meta-analysis of current research. Inf Commun Soc. 2015; 18:524-38. http://dx.doi.org/10.1080/1369118X.2015.1008542.

16. Zappavigna M. Ambient affiliation: a linguistic perspective on twitter. New MediaSoc. 2011; 13:788-806. http://dx.doi.org/10.1177/1461444810385097.

17. Michael, Warner. Publics and counterpublics. Speech. 2002; 88:413-25.

18. Social media tackles controversial issues | pew research center. n.d. http://www.journalism.org/ 2010/02/12/social-media-tackles-controversial-issues/ (accessed June 7, 2016)

19. Twitter reacts to second video exposing Planned Parenthood. n.d. http://dailysignal.com/ 2015/07/21/twitter-reacts-to-another-video-exposing-planned-parenthood/ (accessed November 23, 2016)

20. Hopkins D, King G. A method of automated nonparametric content analysis for social science. Polit Sci. 2010; 54:229-47.

21. Rao, A., Spasojevic, N., Li, Z., DSouza, T. Big data big data 2015 IEEE Int Conf On. IEEE; 2015. Klout score: measuring influence across multiple social networks; p. 2282-9.

22. Anger, I., Kittl, C. Proc 11th Int Conf Knowl Manag Knowl Technol. New York, NY, USA: ACM; 2011. Measuring influence on TWITTER; p. 31:1-4.http://dx.doi.org/10.1145/2024288.2024326

23. Geography UCB. 2010 geographic terms and concepts - census divisions and census regions. n.d. https://www.census.gov/geo/reference/gtc/gtc_census_divreg.html (accessed October 20, 2016)

24. Howard PN, Kollanyi B. Bots,\# strongerin, and\# brexit: computational propaganda during the UKEU referendum. Available SSRN. 2016; 2798311

25. Cogburn DL, Espinoza-Vasquez FK. From networked nominee to networked nation: examining the impact of web 2.0 and social media on political participation and civic engagement in the 2008 Obama campaign. J Polit Mark. 2011; 10:189-213. http://dx.doi.org/ 10.1080/15377857.2011.540224.

26. Stevenson AJ. Finding the twitter users who stood with Wendy. Contraception. 2014; 90:502-7. http://dx.doi.org/10.1016/j.contraception.2014.07.007. [PubMed: 25129330]

27. Borgesius, ZJF., Trilling, D., Moeller, J., Bodó, B., Vreese, D., et al. Should we worry about filter bubbles?. Rochester, NY: Social Science Research Network; 2016.

28. Bakshy E, Messing S, Adamic LA. Exposure to ideologically diverse news and opinion on Facebook. Science. 2015; 348:1130-2. http://dx.doi.org/10.1126/science.aaa1160. [PubMed: 25953820]

29. Pazol K, Creanga AA, Jamieson DJ, Centers for Disease Control and Prevention (CDC). Abortion surveillance - United States 2012. Morb Mortal Wkly Rep Surveill Summ Wash DC. 2002; 64:140. 2015. DOI: $10.15585 / \mathrm{ss} 6410 \mathrm{a} 1$

30. Zane S, Creanga AA, Berg CJ, Pazol K, Suchdev DB, Jamieson DJ, et al. Abortion-related mortality in the United States: 1998-2010. Obstet Gynecol. 2015; 126:258-65. http://dx.doi.org/ 10.1097/AOG.0000000000000945. [PubMed: 26241413]

31. Ruths D, Pfeffer J. Social media for large studies of behavior. Science. 2014; 346:1063-4. http:// dx.doi.org/10.1126/science.346.6213.1063. [PubMed: 25430759]

32. Street 1615 L., NW, Washington S 800, inquiries D 200362024194300 | M 2024194349 | F 202 4194372 |M. Twitter users. Pew res cent internet Sci Tech. 2015. http://www.pewinternet.org/ 2015/01/09/social-media-update-2014/pi_2015-01-09_social-media-new_01/ (accessed July 13, 2016)

33. De Choudhury M, Lin Y-R, Sundaram H, Candan KS, Xie L, Kelliher A, et al. How does the data sampling strategy impact the discovery of information diffusion in social media? ICWSM. 2010; $10: 34-41$.

34. Marres N. Why map issues? On controversy analysis as a digital method. Sci Technol Hum Values. 2015; 40:655-86. http://dx.doi.org/10.1177/0162243915574602.

35. Exploring challenges, progress, and new models for engaging the public in the clinical research enterprise: clinical research roundtable workshop summary. Washington, D.C: National Academies Press; 2003. 
36. North Carolina's anti-LGBT law could cost the state millions in lost revenue | US news | The Guardian. n.d. https://www.theguardian.com/us-news/2016/apr/19/north-carolina-anti-lgbt-lawbathroom-bill-hb2-economic-losses (accessed July 14, 2016)

37. Democrats stage sit-in on house floor to force gun vote. POLITICO. n.d. http://www.politico.com/ story/2016/06/democrats-stage-sit-in-on-house-floor-to-force-gun-vote-224656 (accessed July 14, 2016)

38. Nearly 1 million babies killed by Planned Parenthood last year according to abortion Giant. RedState. n.d. http://www.redstate.com/brandon_morse/2016/07/14/nearly-1-million-babieskilled-planned-parenthood-last-year-according-abortion-giant/ (accessed July 14, 2016) 


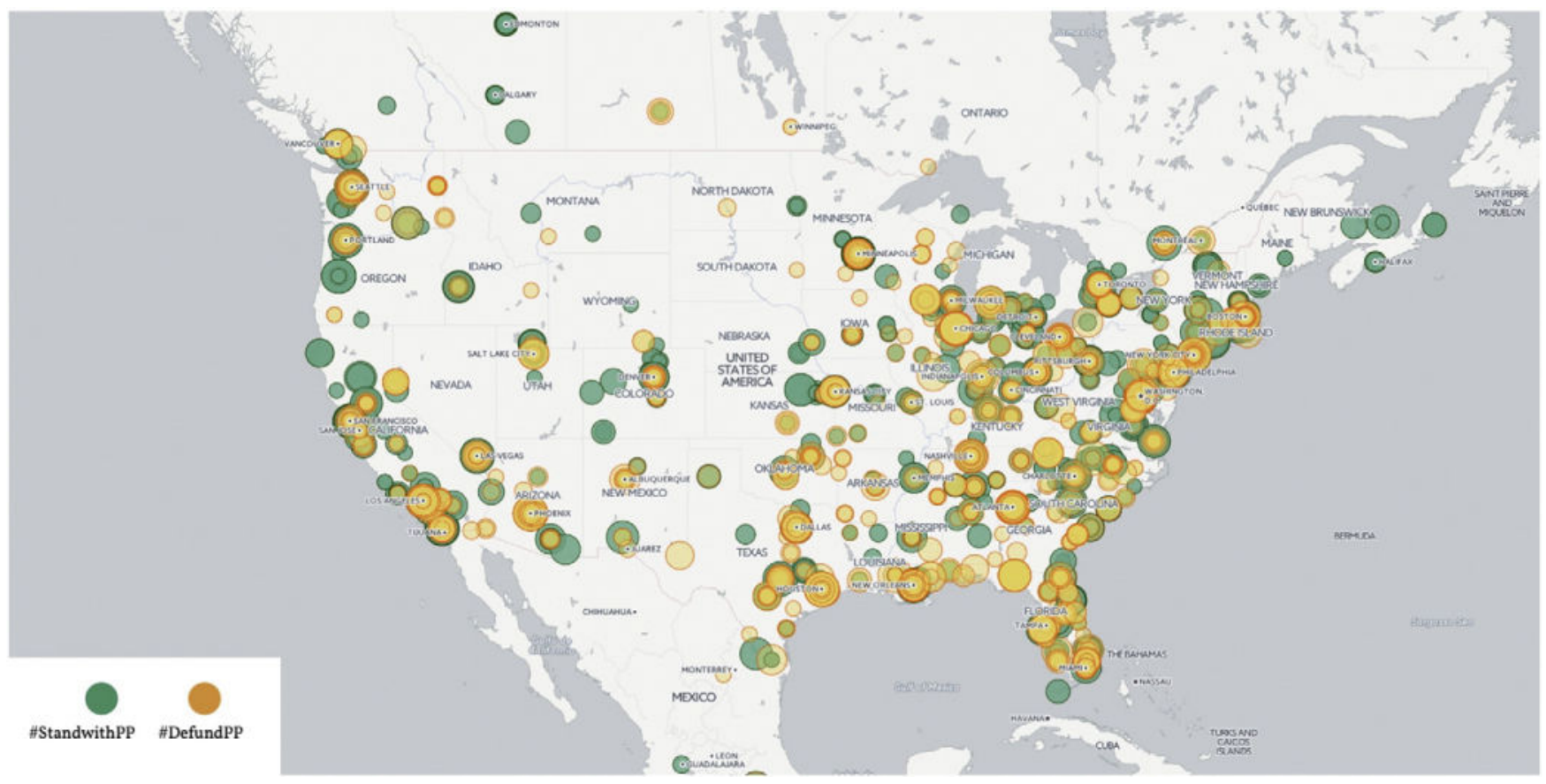

Fig. 1.

Geographic distribution: geographic origin of tweets containing "planned parenthood" sorted by those containing "\#standwithPP" and "\#defundPP." The size of the circle represents the relative Klout score (social influence) of the account. 


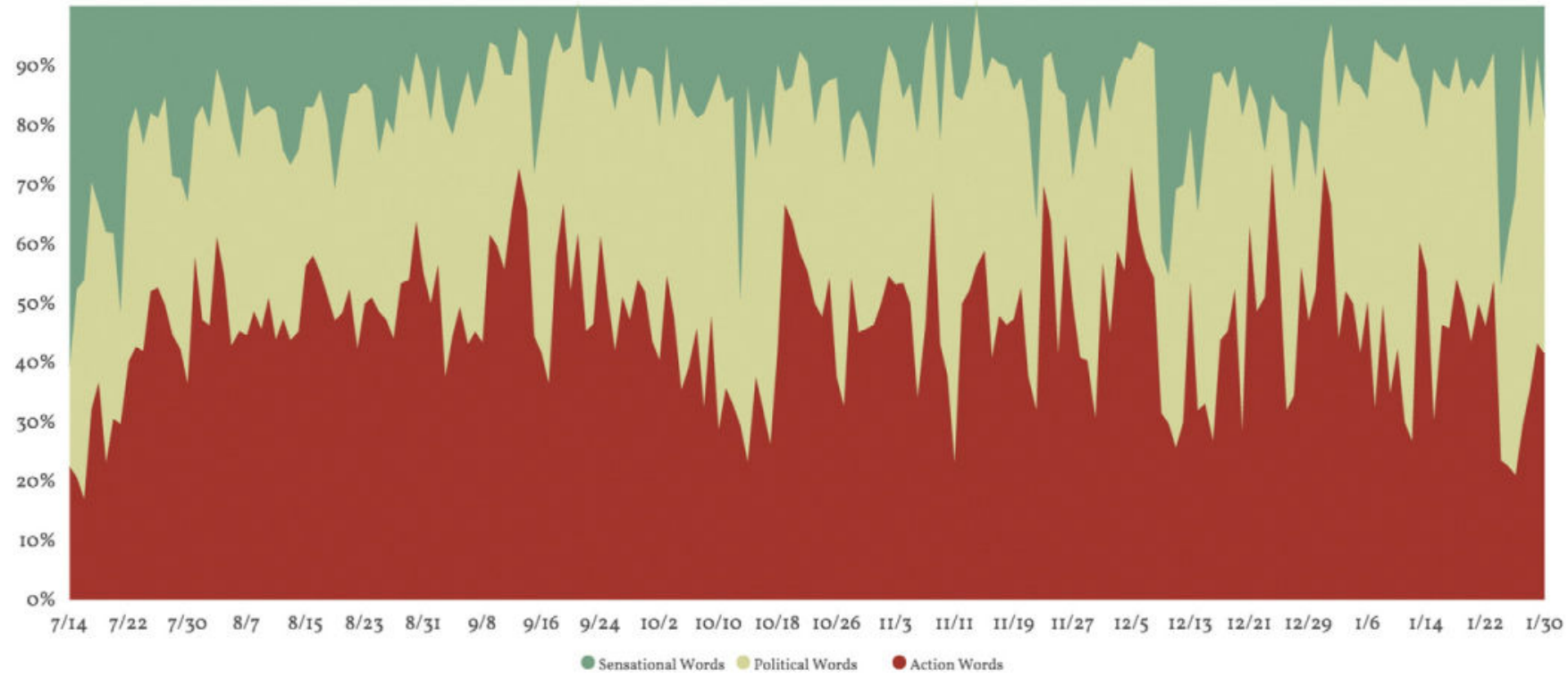

Fig. 2.

Topic distribution: use of "sensational," "political" and "action" words over time in tweets containing "planned parenthood." 


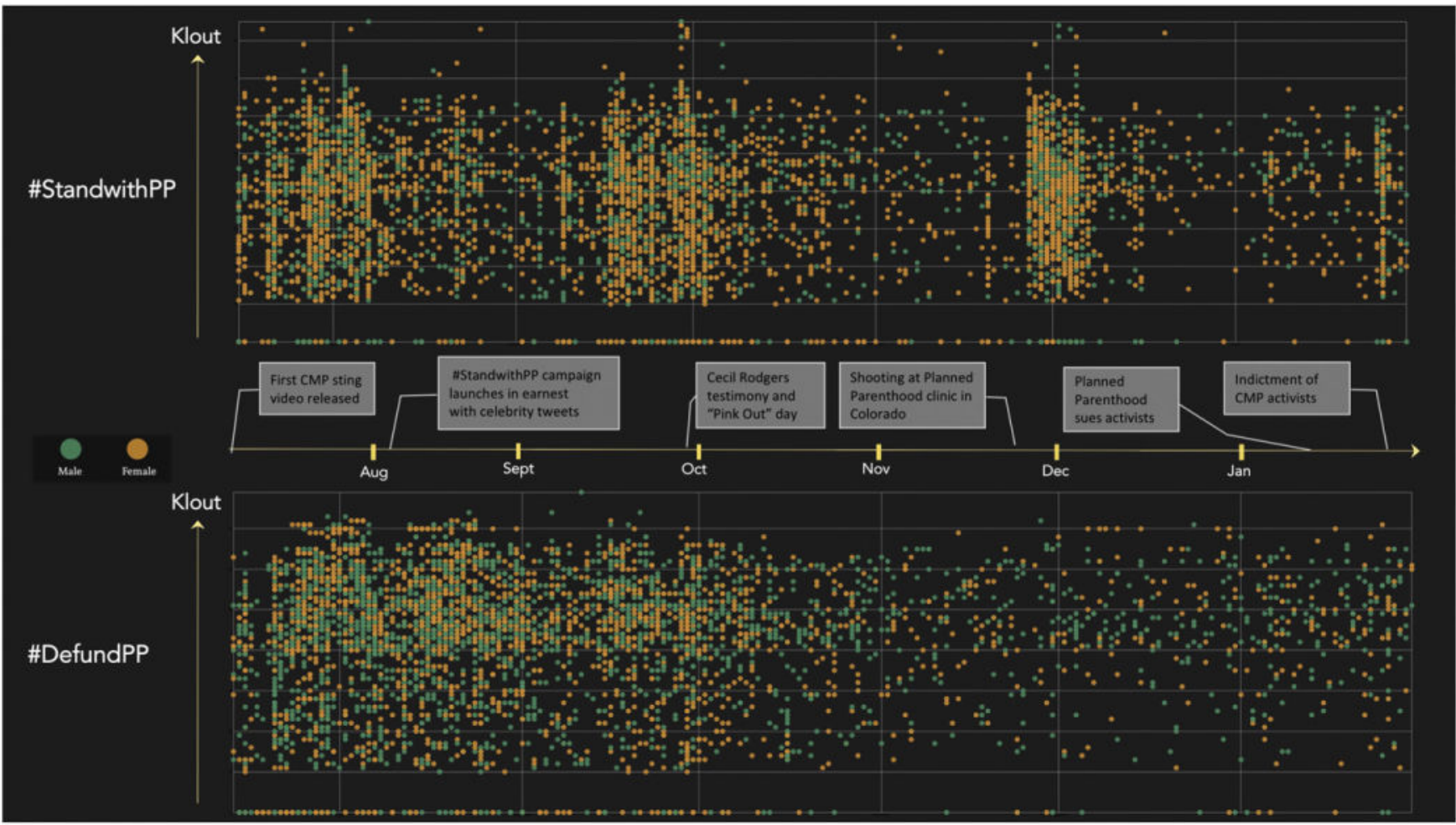

Fig. 3.

Tweets over time: volume of tweets by gender containing "\#standwithPP" and "\#defundPP" over the time period of July 14, 2015, to January 30, 2016. The vertical axis plots the relative Klout score of the user. 


\section{Table 1}

Data categories provided by Twitter Firehose used in this analysis

\begin{tabular}{ll}
\hline Category & Definition \\
\hline Identifier & Twitter database ID \\
Date and time & Timestamp of when tweet was published \\
Link & URL for accessing original tweet \\
Tweet & Tweet text/content \\
Author handle & Username associated with Twitter account \\
Author name & If given by author \\
Geographical location & By country, state/region and city if available \\
Klout score & Measure of social influence (scale 1-100) \\
Gender & If given by author \\
Posts & Number of previous posts author had made up until the time of tweet \\
Followers & Number of other Twitter users following this account at time of tweet \\
Following & Number of accounts this Twitter user is following at time of tweet \\
\hline
\end{tabular}




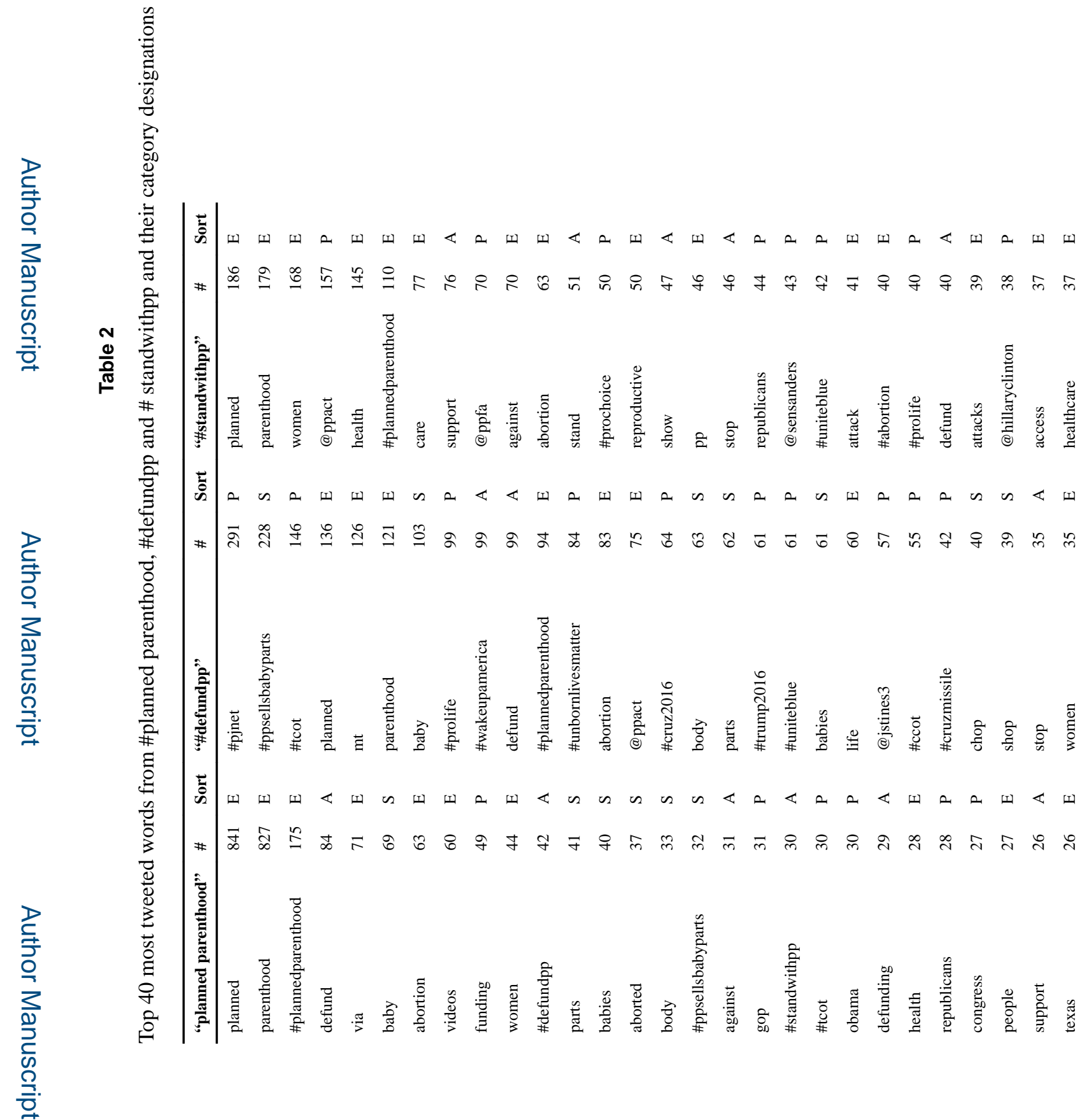




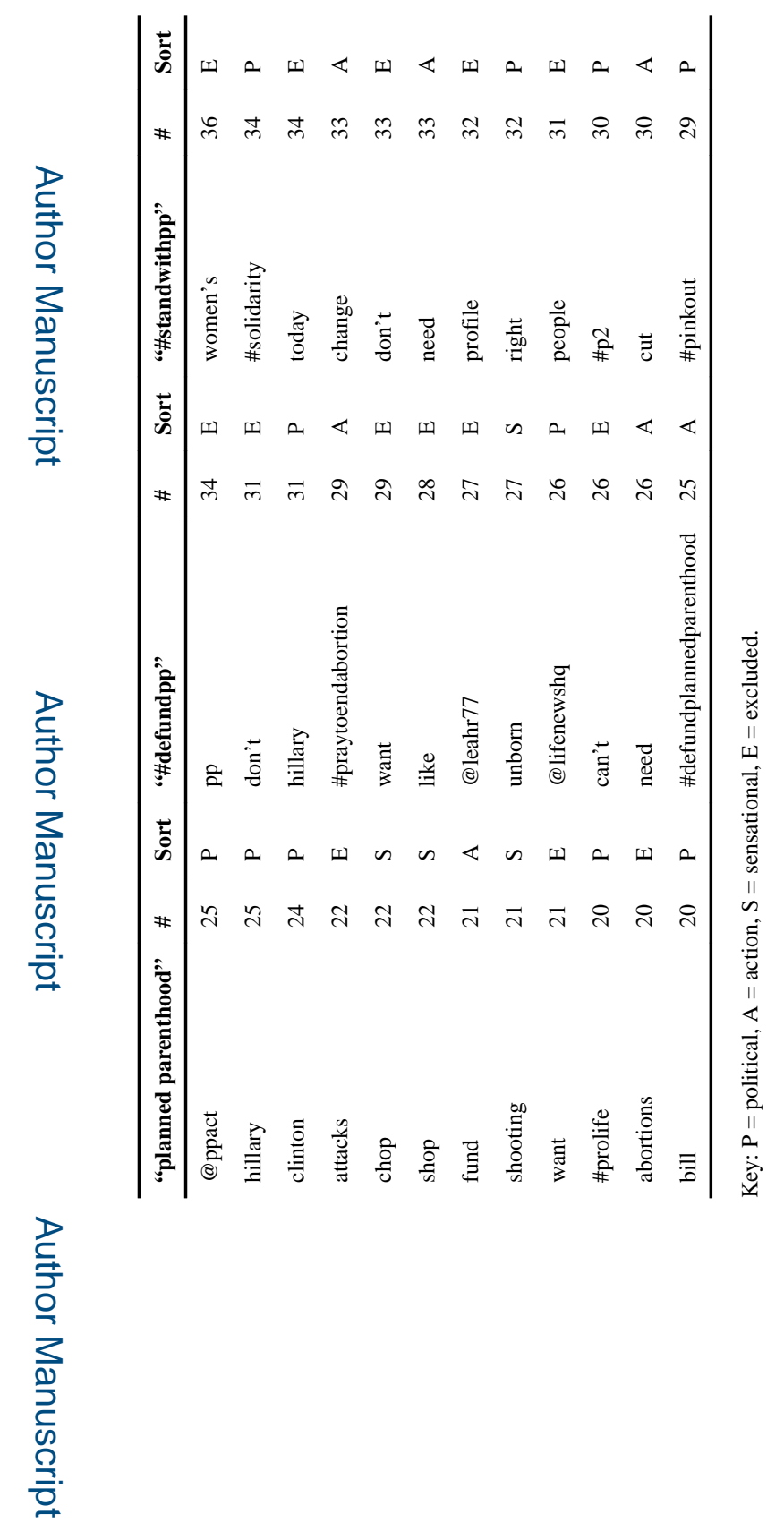

로을 